\title{
Nonempirical density functionals investigated for jellium: Spin-polarized surfaces, spherical clusters, and bulk linear response
}

\author{
Jianmin Tao, ${ }^{1}$ John P. Perdew, ${ }^{2}$ Luís Miguel Almeida, ${ }^{3}$ Carlos Fiolhais, ${ }^{4}$ and Stephan Kümmel ${ }^{5}$ \\ ${ }^{1}$ Theoretical Division and CNLS, Los Alamos National Laboratory, Los Alamos, New Mexico 87545, USA \\ ${ }^{2}$ Department of Physics and Quantum Theory Group, Tulane University, New Orleans, Louisiana 70118, USA \\ ${ }^{3}$ Department of Physics, University of Aveiro, 3810 Aveiro, Portugal \\ ${ }^{4}$ Department of Physics and Center for Computational Physics, University of Coimbra, 3004-516 Coimbra, Portugal \\ ${ }^{5}$ Physics Institute, University of Bayreuth, D-95440 Bayreuth, Germany
}

(Received 8 April 2008; published 5 June 2008)

\begin{abstract}
Jellium, a simple model of metals, is a standard testing ground for density functionals both for bulk and for surface properties. Earlier tests show that the Tao-Perdew-Staroverov-Scuseria (TPSS) nonempirical metageneralized gradient approximation (meta-GGA) for the exchange-correlation energy yields more accurate surface energies than the local spin density (LSD) approximation for spin-unpolarized jellium. In this study, work functions and surface energies of a jellium metal in the presence of "internal" and external magnetic fields are calculated with LSD, Perdew-Burke-Ernzerhof (PBE) GGA, and TPSS meta-GGA and its predecessor, the nearly nonempirical Perdew-Kurth-Zupan-Blaha meta-GGA, using self-consistent LSD orbitals and densities. The results show that (i) For normal bulk densities, the surface correlation energy is the same in TPSS as in PBE, as it should be since TPSS strives to represent a self-correlation correction to PBE; (ii) Normal surface density profiles can be scaled uniformly to the low-density or strong-interaction limit, and TPSS provides an estimate for that limit that is consistent with (but probably more accurate than) other estimates; (iii) For both normal and low densities, TPSS provides the same description of surface magnetism as PBE, suggesting that these approximations may be generally equivalent for magnetism. The energies of jellium spheres with up to 106 electrons are calculated using density functionals and compared to those obtained with diffusion quantum Monte Carlo data, including our estimate for the fixed-node correction. Typically, while PBE energies are too low for spheres with more than about two electrons, LSD and TPSS are accurate there. We confirm that curvature energies are lower in PBE and TPSS than in LSD. Finally, we calculate the linear response of bulk jellium using these density functionals and find that not only LSD but also PBE GGA and TPSS meta-GGA yield a linear response in good agreement with that of the quantum Monte Carlo method, for wave vectors of the perturbing external potential up to twice the Fermi wave vector.
\end{abstract}

DOI: 10.1103/PhysRevB.77.245107 PACS number(s): 71.15.Mb, 31.15.eg, 71.45.Gm, 31.15.ej

\section{INTRODUCTION}

Jellium is a simple model of metals. The surface properties of jellium can emulate those of real surfaces. In this popular model, the lattice of ions is replaced by a uniform positive charge density. Lang and Kohn ${ }^{1}$ made the first selfconsistent calculation of the surface properties of this model using the local spin density (LSD) approximation ${ }^{2}$ for the exchange-correlation (xc) energy within the Kohn-Sham density functional formalism. ${ }^{2-4}$ The results, after a correction for lattice effects, agreed surprisingly well with experiments. This unexpected success initiated the application of the density functional theory to surfaces. A restricted variational calculation by Mahan ${ }^{5}$ gave density profiles and surface energies very similar to those of Lang and Kohn. ${ }^{1}$

Perdew and Monnier ${ }^{6-8}$ performed a series of selfconsistent calculations of the surface properties of real and jellium metals within LSD. Kautz and Schwartz ${ }^{9}$ extended the work of Lang and Kohn ${ }^{1}$ to the spin-polarized case and calculated self-consistently several surface properties of jellium polarized by a magnetic field uniform inside and zero outside the edge of the positive background. Over the past few decades, the correct surface energy of jellium has been controversial. Recent work ${ }^{10-16}$ resolves most of the controversy, placing this energy close to but probably a little higher than that of LSD (which benefits from a strong error cancellation between exchange and correlation). For a review of this and earlier work, see Ref. 16.

In density functional theory, ${ }^{2-4}$ everything is treated exactly except the xc energy, which has to be approximated as a functional of the electron density. The development of better density functional approximations has been the subject of continuing theoretical efforts. LSD is the simplest approximation and has been successful in condensed matter physics. However, it tends to overbind molecules. An efficient way to solve this problem is to introduce the density gradient $\nabla n$ as an additional local ingredient to construct a gradientcorrected density functional, the generalized gradient approximation (GGA). ${ }^{17,18}$ With the advent of GGA, density functional theory has become a popular method in quantum chemistry as well. Although GGAs have achieved significant improvement over LSD for most properties and for diverse systems, ${ }^{19}$ they usually underestimate the surface exchangecorrelation energy of a spin-unpolarized jellium, for which LSD is much more accurate. ${ }^{11,20}$

Further systematic improvement over GGAs may be made by imposing additional exact conditions without losing those already satisfied by GGA. This can be done by employing the kinetic energy densities $\tau_{\uparrow}(\mathbf{r})$ and $\tau_{\downarrow}(\mathbf{r})$ and/or the Laplacians of the densities $\nabla^{2} n_{\uparrow}(\mathbf{r})$ and $\nabla^{2} n_{\downarrow}(\mathbf{r})$ as further 
additional ingredients. Here, the kinetic energy density of electrons with spin $\sigma(\sigma=\uparrow, \downarrow)$ is defined as

$$
\tau_{\sigma}(\mathbf{r})=\frac{1}{2} \sum_{i}^{\text {occup }}\left|\nabla \psi_{i}^{\sigma}(\mathbf{r})\right|^{2},
$$

where $\psi_{i}^{\sigma}(\mathbf{r})$ are the occupied Kohn-Sham orbitals, which are implicit functionals of the density $n_{\sigma}(\mathbf{r})$. (Atomic units $\hbar=m=e^{2}=1$ are used throughout unless otherwise explicitly stated.) This family of density functional approximations is called the meta-GGA. ${ }^{21-23}$

While the exact form of the universal functional remains unknown, many exact constraints on this exact functional have been uncovered. Thus, the more exact constraints a density functional satisfies, the closer it is to the exact universal functional. Having this in mind, Perdew-KurthZupan-Blaha (PKZB) ${ }^{22}$ constructed a meta-GGA from first principles. This nearly nonempirical functional satisfies two important constraints which cannot be satisfied at the GGA level: It nearly recovers the known fourth-order gradient expansion $^{24}$ of the exchange energy in the slowly varying limit and it is free from self-correlation errors.

PKZB meta-GGA has impressively corrected ${ }^{11,20,22}$ the too-low surface exchange-correlation energy of GGA functionals and has successfully improved upon LSD and GGAs in thermochemistry for molecular atomization energies. ${ }^{25}$ The defect of PKZB is that it contains an empirical parameter in its exchange term, which was fitted to molecular atomization energies. Consequently, PKZB produces too-long bond lengths and some inaccurate properties of hydrogenbonded complexes. ${ }^{19,25,26}$ These failures may be attributed to the unbalanced description of PKZB exchange and correlation for slowly varying densities and one- or two-electron densities, which are the paradigms in condensed matter physics and in quantum chemistry, respectively.

Starting with the Perdew-Burke-Ernzerhof (PBE) GGA, Tao-Perdew-Staroverov-Scuseria $(\text { TPSS })^{23}$ constructed a nonempirical meta-GGA. While the formula for TPSS looks a little more complicated than the PKZB one, the guiding theory is simple. A sound meta-GGA should be able to describe well the paradigm densities of condensed matter physics and quantum chemistry. By imposing correct constraints, a meta-GGA can be made accurate for diverse systems of interest. The TPSS construction builds many additional correct constraints ${ }^{11,27}$ into a meta-GGA while retaining those that the GGA has already respected. As a result, this metaGGA is uniformly accurate for various properties of diverse systems, ${ }^{28}$ suggesting the correctness of the TPSS philosophy.

For high-density (exchange dominated) systems such as atoms, the TPSS is remarkably accurate. ${ }^{27,29}$ In the lowdensity or strong-interaction limit, TPSS recovers the PKZB correlation, which is accurate for spin-unpolarized densities. The relative spin polarization is defined as

$$
\zeta=\frac{n_{\uparrow}-n_{\downarrow}}{n},
$$

where $n=n_{\uparrow}+n_{\downarrow}$. Since TPSS correlation strives to represent a self-correlation correction to PBE, it should not change
PBE correlation for a system with delocalized electrons, whether spin-polarized or not. This requirement is satisfied by TPSS through the design for a spin-unpolarized jellium. ${ }^{11}$ However, we never impose this requirement for a spinpolarized density. Instead, we first scale to the low-density limit $^{30-33}$ and there require ${ }^{27}$ the exchange-correlation energy to be correctly independent of spin for a model uniformly spin-polarized one-electron Gaussian density with constant relative spin polarization in the range of $0 \leq|\zeta| \leqslant 0.7$, like LSD and PBE and unlike PKZB.

In this work, we investigate the spin dependence of TPSS correlation and find that it is nearly the same as that of the PBE for spin-polarized jellium generated by magnetic fields, implying that TPSS does not alter the PBE correlation energy for a spin-polarized system with delocalized electrons. Since TPSS successfully improves on LSD for spin-unpolarized jellium and has the proper spin dependence, we estimate the surface exchange-correlation energy and work function with the TPSS meta-GGA functional for a spin-polarized jellium in magnetic fields. An application to the infinite barrier model $^{34}$ of metal surfaces is a related test but for rapidly varying densities. The other tests considered here are the jellium spheres (which sample the surface and curvature energy) and the linear response of the bulk jellium.

\section{DENSITY FUNCTIONAL APPROXIMATIONS}

Density functionals may be ordered by the "Jacob's ladder" 35,36 of approximations, according to the type of their local ingredients, whether constructed nonempirically or empirically. Here, we only focus on the all-purpose nonempirical functionals, LSD, PBE, TPSS, and the nearly nonempirical PKZB, but not the ones recently developed for solids and solid surfaces such as AM05, ${ }^{37} \mathrm{Wu}-\mathrm{Cohen},{ }^{38}$ and PBEsol. ${ }^{39}$ The first three rungs of the ladder to be considered here are LSD, PBE, and TPSS. (Note that the GGA described in Ref. 38 is constructed in part from TPSS by the approximation $\tilde{q}_{b} \approx 2 p / 3$ for slowly varying densities, which is a misinterpretation of a statement in Ref. 23; it is only for $\alpha=1$, as in the uniform gas, and not for $\alpha \approx 1$, that $\tilde{q}_{b} \approx 2 p / 3$.)

Because the exchange component of a density functional satisfies the spin scaling relation, ${ }^{40}$

$$
E_{\mathrm{x}}\left[n_{\uparrow}, n_{\downarrow}\right]=E_{x}\left[2 n_{\uparrow}\right] / 2+E_{\mathrm{x}}\left[2 n_{\downarrow}\right] / 2,
$$

where $E_{\mathrm{x}}[n] \equiv E_{\mathrm{x}}[n / 2, n / 2]$ and $n(\mathbf{r})=n_{\uparrow}(\mathbf{r})+n_{\downarrow}(\mathbf{r})$, we only need an exchange functional $E_{\mathrm{x}}[n]$ of a spin-unpolarized system. An exchange functional also satisfies the uniform coordinate scaling requirement ${ }^{41}$

$$
E_{\mathrm{x}}\left[n_{\gamma}\right]=\gamma E_{\mathrm{x}}[n],
$$

where $n_{\gamma}=\gamma^{3} n(\gamma \mathbf{r})$ is the scaled density of $n(\mathbf{r})$. These two constraints are the basic requirements of an exchange functional.

For a spin-unpolarized (closed-shell) system, the exchange functionals of the first three rungs may be expressed in the form 


$$
E_{\mathrm{x}}[n]=\int d^{3} r n \epsilon_{\mathrm{x}}^{\mathrm{unif}}(n) F_{\mathrm{x}}
$$

where $\epsilon_{\mathrm{x}}^{\text {unif }}(n)=-\frac{3}{4 \pi}\left(3 \pi^{2} n\right)^{1 / 3}$ is the exchange energy per particle of a uniform electron gas and $F_{\mathrm{x}}$ is the exchange enhancement factor showing the nonlocality, ${ }^{27}$

$$
F_{\mathrm{x}}=1+\kappa-\kappa /(1+x / \kappa),
$$

with $\kappa=0.804$ and $x \geq 0$. The order of the ladder rungs depends upon the choice of $x$ in Eq. (6). We have $x=0$ for LSD and $x=\mu p$ for PBE, where $\mu=0.21951$, and

$$
p=|\nabla n|^{2} /\left[4\left(3 \pi^{2}\right)^{2 / 3} n^{8 / 3}\right]=s^{2}
$$

is the square of the reduced density gradient $s$. For the TPSS and PKZB meta-GGAs, $x$ is a function of the two variables $p$ and $z$, where

$$
z=\tau^{W} / \tau \leq 1,
$$

with $\tau=\Sigma_{\sigma} \tau_{\sigma}$ and with $\tau^{W}=\frac{1}{8}|\nabla n|^{2} / n$ being the von Weizsäcker kinetic energy density. In the uniform-gas limit, all the density functionals above the first rung correctly reduce to LSD. This uniform-gas limit is the most important requirement ${ }^{42}$ for bulk solids and surfaces.

Since the correlation component of a density functional does not have such a simple spin scaling relation as the exchange component, we have to build the spin dependence into the correlation part. The LSD correlation energy has the form

$$
E_{\mathrm{c}}^{\mathrm{LSD}}\left[n_{\uparrow}, n_{\downarrow}\right]=\int d^{3} r n \epsilon_{\mathrm{c}}^{\mathrm{unif}}\left(n_{\uparrow}, n_{\downarrow}\right),
$$

where $\epsilon_{\mathrm{c}}^{\text {unif }}$ is the correlation energy per electron ${ }^{43}$ for a uniform electron gas. The PBE correlation ${ }^{18,44}$ is based on the LSD correlation,

$$
E_{\mathrm{c}}^{\mathrm{PBE}}\left[n_{\uparrow}, n_{\downarrow}\right]=\int d^{3} r n \epsilon_{\mathrm{c}}^{\mathrm{PBE}}\left(n_{\uparrow}, n_{\downarrow}, \nabla n_{\uparrow}, \nabla n_{\downarrow}\right),
$$

where $\epsilon_{\mathrm{c}}^{\mathrm{PBE}}$ correctly reduces to $\epsilon_{\mathrm{c}}^{\text {unif }}$ in the uniform-gas limit. The TPSS correlation ${ }^{23,27}$ is constructed from the PBE correlation,

$$
E_{\mathrm{c}}^{\mathrm{TPSS}}\left[n_{\uparrow}, n_{\downarrow}\right]=\int d^{3} r n \epsilon_{\mathrm{c}}^{\mathrm{TPSS}}\left(n_{\uparrow}, n_{\downarrow}, \nabla n_{\uparrow}, \nabla n_{\downarrow}, \tau_{\uparrow}, \tau_{\downarrow}\right),
$$

where

$$
\epsilon_{\mathrm{c}}^{\mathrm{TPSS}}=\epsilon_{\mathrm{c}}^{\mathrm{rev}-\mathrm{PKZB}}\left[1+d \epsilon_{\mathrm{c}}^{\mathrm{rev}-\mathrm{PKZB}}\left(\tau^{W} / \tau\right)^{3}\right] .
$$

The quantity $\epsilon_{\mathrm{c}}^{\mathrm{rev}-\mathrm{PKZB}}$ of Eq. (12) is the revised PKZB correlation,

$$
\begin{aligned}
\epsilon_{\mathrm{c}}^{\mathrm{rev}-\mathrm{PKZB}}= & \epsilon_{\mathrm{c}}^{\mathrm{PBE}}\left(n_{\uparrow}, n_{\downarrow}, \nabla n_{\uparrow}, \nabla n_{\downarrow}\right)\left[1+C(\zeta, \xi)\left(\tau^{W} / \tau\right)^{2}\right] \\
& -[1+C(\zeta, \xi)]\left(\tau^{W} / \tau\right)^{2} \sum_{\sigma} \frac{n_{\sigma}}{n} \widetilde{\epsilon}_{\mathrm{c}}^{\sigma},
\end{aligned}
$$

with $\widetilde{\epsilon}_{\mathrm{c}}^{\sigma}$ being

$$
\widetilde{\epsilon}_{\mathrm{c}}^{\sigma}=\max \left[\epsilon_{\mathrm{c}}^{\mathrm{PBE}}\left(n_{\sigma}, 0, \nabla n_{\sigma}, 0\right), \epsilon_{\mathrm{c}}^{\mathrm{PBE}}\left(n_{\uparrow}, n_{\downarrow}, \nabla n_{\uparrow}, \nabla n_{\downarrow}\right)\right],
$$

where $\zeta$ is the relative spin polarization defined as $\zeta(\mathbf{r})$ $=\left[n_{\uparrow}(\mathbf{r})-n_{\downarrow}(\mathbf{r})\right] / n(\mathbf{r})$ and $\xi=|\nabla \zeta| /\left[2\left(3 \pi^{2} n\right)^{1 / 3}\right] .{ }^{23,27,45}$ Here, $C(\zeta, \xi)$ at $\zeta=0$ and $\xi=0$ in Eq. (13) is chosen so that, in the low-density or strong-interaction limit, TPSS correlation recovers PKZB correlation, which is accurate ${ }^{27,33}$ for spinunpolarized densities. The parameter $d$ in Eq. (12) is chosen such that the self-interaction correction should not alter the surface PBE correlation energy for spin-unpolarized jellium with delocalized electrons. The natural construction of the spin-dependent $C(\zeta, \xi)$ would be to make the TPSS correlation remain the same as the $\mathrm{PBE}$ correlation for spinpolarized jellium in the achievable (and thus energetically important) range of the uniform bulk relative spin polarization $0 \leq \zeta \leqq 0.7$. However, $C(\zeta, \xi)$ is designed instead to make TPSS independent of spin in the low-density limit for the one-electron Gaussian density and other densities with uniform $\zeta$ in the range of $0 \leq \zeta \leqq 0.7$ without changing other properties. We will show that these two different procedures are essentially equivalent in the construction of the spindependent parameter $C(\zeta, \xi)$.

\section{SPIN-POLARIZED JELLIUM AND KOHN-SHAM APPROACH}

In a semi-infinite jellium metal filling the half space $x$ $<0$, the uniform positive background density corresponding to the ion lattice may be written as ${ }^{1}$

$$
n_{+}(\mathbf{r})=\bar{n} \theta(-x)
$$

where $\theta(-x)$ is a step function and $\bar{n}$ is the bulk electron density. In the absence of an external electric field, the electron density $n(\mathbf{r})$ is related to the background density via the charge neutralization condition ${ }^{7,9}$

$$
\int d^{3} r\left[n(\mathbf{r})-n_{+}(\mathbf{r})\right]=0 .
$$

In the presence of an external magnetic field $\mathbf{B}(\mathbf{r})$, the self-consistent single-particle Kohn-Sham equation may be written as 9

$$
\left\{-\frac{1}{2} \nabla^{2}+v_{\mathrm{eff}}^{\sigma}(\mathbf{r})\right\} \psi_{i}^{\sigma}(\mathbf{r})=\epsilon_{i}^{\sigma} \psi_{i}^{\sigma}(\mathbf{r}),
$$

where $v_{\text {eff }}^{\sigma}$ is the local effective potential given by

$$
v_{\mathrm{eff}}^{\sigma}(\mathbf{r})=v_{\mathrm{ext}}(\mathbf{r})+\int d^{3} r^{\prime} \frac{n\left(\mathbf{r}^{\prime}\right)}{\left|\mathbf{r}^{\prime}-\mathbf{r}\right|}-\boldsymbol{\sigma} \cdot \mu_{B} \mathbf{B}(\mathbf{r})+v_{\mathrm{xc}}^{\sigma}(\mathbf{r}) .
$$

Here, $\boldsymbol{\sigma} \cdot \mathbf{B}=\sigma B$, with $\sigma= \pm 1, \mu_{B}=e \hbar / 2 m$ is the Bohr magneton, $v_{\mathrm{xc}}^{\sigma}(\mathbf{r})=\delta E_{\mathrm{xc}} / \delta n_{\sigma}(\mathbf{r})$ is the exchange-correlation potential, and $v_{\text {ext }}(\mathbf{r})$ is the external scalar potential,

$$
v_{\text {ext }}(\mathbf{r})=-\int d^{3} r^{\prime} \frac{n_{+}\left(\mathbf{r}^{\prime}\right)}{\left|\mathbf{r}^{\prime}-\mathbf{r}\right|}
$$

Here, $\sigma=+1$ corresponds to electrons with spin $\sigma$ parallel to $\mathbf{B}$ and -1 to electrons with spin $\sigma$ antiparallel to $\mathbf{B}$. The 
electron density $n_{\sigma}$ may be evaluated from the occupied Kohn-Sham orbitals via

$$
n_{\sigma}(\mathbf{r})=\sum_{i}\left|\psi_{i}^{\sigma}\right|^{2} \theta\left(\epsilon_{F}^{\sigma}-\epsilon_{i}^{\sigma}\right)
$$

where $\epsilon_{F}^{\sigma}=k_{F}^{\sigma 2} / 2$, with $k_{F}^{\sigma}=\left(6 \pi^{2} n_{\sigma}\right)^{1 / 3}$ is the Fermi energy per electron of spin $\sigma$. Since $v_{\text {eff }}^{\sigma}$ of Eq. (17) depends upon the density $n_{\sigma}$ of Eq. (20), Eqs. (17)-(20) must be solved selfconsistently.

Suppose the external magnetic field is uniform for $x<0$ (within the bulk metal). Then, the bulk density is spinpolarized uniformly with position-independent relative spin polarization. At the Fermi levels, the spin-up and spin-down electrons have the same chemical potential,

$$
\mu_{\uparrow}=\mu_{\downarrow},
$$

where

$$
\mu_{\sigma}=\delta E / \delta n_{\sigma}(\mathbf{r})=\frac{\delta T_{s}}{\delta n_{\sigma}(\mathbf{r})}+v_{\mathrm{ext}}(\mathbf{r})+u_{H}(\mathbf{r})-\sigma \mu_{B} B+v_{\mathrm{xc}}^{\sigma}(\mathbf{r}) .
$$

Here, $T_{s}\left[n_{\uparrow}, n_{\downarrow}\right]$ is the Kohn-Sham kinetic energy evaluated via Eq. (1) and $u_{H}$ is the Hartree potential, which is given by the second term of Eq. (18). The bulk uniform relative spin polarization is determined by the strength $\mu_{B} B$ of the applied external magnetic field via the relation

$$
\mu_{B} B=\left(\epsilon_{F}^{\uparrow}-\epsilon_{F}^{\downarrow}\right) / 2+\left[v_{\mathrm{xc}}^{\uparrow}\left(r_{s}, \zeta\right)-v_{\mathrm{xc}}^{\downarrow}\left(r_{s}, \zeta\right)\right] / 2,
$$

where $r_{s}=(3 / 4 \pi \bar{n})^{1 / 3}$ is the bulk Seitz radius. For convenience, we set the spin-up and spin-down Fermi levels at zero energy, so that we have

$$
v_{\mathrm{eff}}^{\sigma}(\mathbf{r})=-\frac{\delta T_{s}}{\delta n_{\sigma}(\mathbf{r})} .
$$

Because the positive background is uniform, the electron density is also uniform in the bulk. The Kohn-Sham wave functions as solutions of one-electron Kohn-Sham equation (17) are plane waves and $v_{\text {eff }}^{\sigma}(-\infty)=-\epsilon_{F}^{\sigma}$. The electrostatic potential is thus given as

$$
v_{e s}(-\infty)=-\epsilon_{F}^{\sigma}-v_{\mathrm{xc}}^{\sigma}(\bar{n}, \zeta)+\sigma \mu_{B} B,
$$

where $v_{e s}=v_{\text {ext }}+u_{H}$.

Near or at the surface, we may write ${ }^{1,9}$

$$
\psi_{i}^{\sigma}(\mathbf{r})=\psi_{k}^{\sigma}(x) e^{i\left(k_{y} y+k_{z} z\right)},
$$

where $k, k_{y}$, and $k_{z}$ are the magnitudes of the wave vectors along the $x, y$, and $z$ directions, respectively. The threedimensional Kohn-Sham equation (17) reduces then to the one-dimensional one ${ }^{1}$ for $\psi_{k}^{\sigma}(x)$,

$$
\left\{-\frac{1}{2} \frac{d^{2}}{d x^{2}}+v_{\mathrm{eff}}^{\sigma}(x)-v_{\mathrm{eff}}^{\sigma}(-\infty)\right\} \psi_{k}^{\sigma}(x)=\epsilon_{k} \psi_{k}^{\sigma}(x),
$$

where $\epsilon_{k}=k^{2} / 2$. Solving Eq. (27) for $\psi_{k}^{\sigma}(x)$ yields the density $n_{\sigma}$ via

$$
n_{\sigma}(x)=3 \bar{n}_{\sigma} \int_{0}^{1} d \tilde{k}\left(1-\widetilde{k}^{2}\right)\left|\psi_{k}^{\sigma}(x)\right|^{2},
$$

where $0 \leq \tilde{k}=k / k_{F}^{\sigma} \leq 1$. In the vacuum, the density decays exponentially, ${ }^{8}$ as in an atom, ${ }^{46}$

$$
\psi_{k}^{\sigma}(x) \rightarrow e^{-a x}, \quad(x \rightarrow \infty),
$$

where $a$ is a constant for a given $\bar{n}$.

Within LSD, the exchange-correlation potential ${ }^{43}$ may be evaluated as

$$
v_{\mathrm{xc}}^{\sigma}=\frac{\partial}{\partial n_{\sigma}}\left[n \epsilon_{\mathrm{xc}}\left(n_{\uparrow}, n_{\downarrow}\right)\right] .
$$

Following Monnier and Perdew ${ }^{8}$ for the treatment of the electrostatic potential and the self-consistency procedure (outlined in Appendix A of Ref. 8), we solved the onedimensional Kohn-Sham equation (27) self-consistently within the LSD.

In the present work, two external magnetic fields coupled to the electron spins are considered: (1) uniform inside and zero outside the jellium edge,

$$
B(x)=B_{0} \theta(-x),
$$

and (2) uniform everywhere,

$$
B(x)=B_{0} .
$$

Equation (31) was proposed by Kautz and Schwartz $^{9}$ to simulate, within the jellium model, the "internal" magnetic field near the surface of a ferromagnetic metal, while Eq. (32) can be realized experimentally over a range of $B_{0}$. The magnitude of the external magnetic fields $B_{0}$ may be found from Eq. (23) for a given $\zeta$.

The work function $W$ (Refs. 1, 8, and 9) is an interesting quantity which can be measured experimentally. It is defined as the energy required to remove an electron from a bulk solid into the vacuum. Within the framework of the KohnSham density functional theory, the work function is the difference of the Kohn-Sham single-particle energies of an electron at rest in the vacuum and an electron moving at the Fermi level in the bulk, ${ }^{8}$ i.e.,

$$
W=v_{\text {eff }}^{\sigma}(\infty)-\left[k_{F}^{\sigma 2} / 2+v_{\text {eff }}^{\sigma}(-\infty)\right] .
$$

In the presence of an external magnetic field, the work function is given by 9

$$
\begin{aligned}
W & =v_{\mathrm{eff}}^{\sigma}(\infty)-\left[k_{F}^{\sigma 2} / 2+v_{\mathrm{eff}}^{\sigma}(-\infty)\right]+\sigma \mu_{B} B \\
& =\left[v_{e s}(\infty)-v_{e s}(-\infty)\right]-v_{\mathrm{xc}}^{\sigma}\left(\bar{n}_{\uparrow}, \bar{n}_{\downarrow}\right)-k_{F}^{\sigma 2} / 2+\sigma \mu_{B} B,
\end{aligned}
$$

where the second equality can be obtained by combining Eqs. (18) and (25).

\section{SURFACE ENERGIES OF SPIN-POLARIZED JELLIUM}

\section{A. Surface energies}

The surface energy of a solid is the energy required to split the solid per unit area of new surface formed. Here, we 
TABLE I. The strength $\mu_{B} B_{0}$ of the two model external magnetic fields of Eqs. (31) and (32), work function $W$, and surface correlation and exchange-correlation energies of the planar jellium surface in LSD, PBE, and PKZB and TPSS, as functions of uniform bulk relative spin polarization $\zeta$ at normal bulk densities $r_{s}=2,4,6 . \mu_{B} B_{0}$ and $W$ are in $\mathrm{eV}$; surface energies are in $\mathrm{erg} / \mathrm{cm}^{2}$. LSD orbitals and densities are used $\left(1\right.$ hartree $=27.21 \mathrm{eV} ; 1$ hartree $\left./ \mathrm{bohr}^{2}=1.557 \times 10^{6} \mathrm{erg} / \mathrm{cm}^{2}\right)$.

\begin{tabular}{|c|c|c|c|c|c|c|c|c|c|c|c|}
\hline \multirow[b]{2}{*}{$r_{s}$} & \multirow[b]{2}{*}{$\zeta$} & \multirow[b]{2}{*}{$\mu_{B} B_{0}$} & \multirow[b]{2}{*}{$W$} & \multicolumn{4}{|c|}{$\sigma_{c}$} & \multicolumn{4}{|c|}{$\sigma_{\mathrm{xc}}$} \\
\hline & & & & LSD & PBE & PKZB & TPSS & LSD & PBE & PKZB & TPSS \\
\hline \multicolumn{12}{|c|}{$B(\mathbf{r})=B_{0} \theta(-x)$} \\
\hline \multirow{7}{*}{2} & 0.0 & 0.0 & 3.80 & 317 & 827 & 824 & 827 & 3354 & 3264 & 3401 & 3380 \\
\hline & 0.2 & 1.29 & 3.78 & 316 & 823 & 822 & 822 & 3350 & 3264 & 3404 & 3380 \\
\hline & 0.4 & 2.59 & 3.75 & 312 & 811 & 818 & 808 & 3337 & 3262 & 3410 & 3377 \\
\hline & 0.6 & 3.95 & 3.68 & 301 & 785 & 810 & 780 & 3315 & 3246 & 3414 & 3360 \\
\hline & 0.8 & 5.44 & 3.54 & 275 & 731 & 794 & 724 & 3268 & 3181 & 3390 & 3294 \\
\hline & 0.0 & 0.0 & 2.91 & 39 & 124 & 124 & 124 & 262 & 253 & 266 & 266 \\
\hline & 0.2 & 0.26 & 2.91 & 40 & 123 & 124 & 123 & 261 & 252 & 267 & 265 \\
\hline \multirow[t]{5}{*}{4} & 0.4 & 0.53 & 2.90 & 41 & 119 & 123 & 118 & 258 & 251 & 270 & 264 \\
\hline & 0.6 & 0.81 & 2.88 & 44 & 112 & 123 & 110 & 252 & 249 & 274 & 260 \\
\hline & 0.8 & 1.12 & 2.87 & 47 & 100 & 124 & 98 & 243 & 241 & 280 & 254 \\
\hline & 0.0 & 0.0 & 2.34 & 10 & 40 & 40 & 40 & 54 & 52 & 55 & 55 \\
\hline & 0.2 & 0.10 & 2.32 & 11 & 39 & 40 & 39 & 53 & 51 & 55 & 55 \\
\hline \multirow[t]{3}{*}{6} & 0.4 & 0.20 & 2.31 & 12 & 38 & 40 & 38 & 52 & 51 & 56 & 54 \\
\hline & 0.6 & 0.30 & 2.30 & 14 & 35 & 41 & 35 & 50 & 49 & 58 & 52 \\
\hline & 0.8 & 0.43 & 2.29 & 17 & 32 & 43 & 32 & 47 & 47 & 61 & 51 \\
\hline \multicolumn{12}{|c|}{$B(\mathbf{r})=B_{0}$} \\
\hline \multirow{4}{*}{2} & 0.2 & 1.29 & 4.21 & 395 & 783 & 794 & 783 & 3363 & 3469 & 3620 & 3586 \\
\hline & 0.4 & 2.59 & 5.20 & 578 & 706 & 758 & 705 & 3384 & 3883 & 4074 & 4001 \\
\hline & 0.6 & 3.95 & 6.46 & 782 & 627 & 733 & 636 & 3466 & 4324 & 4571 & 4454 \\
\hline & 0.8 & 5.44 & 7.93 & 907 & 565 & 715 & 591 & 3734 & 4705 & 4999 & 4853 \\
\hline \multirow{3}{*}{4} & 0.2 & 0.26 & 2.95 & 46 & 120 & 121 & 119 & 261 & 260 & 276 & 273 \\
\hline & 0.4 & 0.53 & 3.06 & 64 & 110 & 119 & 108 & 258 & 277 & 300 & 289 \\
\hline & 0.6 & 0.81 & 3.26 & 86 & 98 & 118 & 97 & 260 & 299 & 335 & 312 \\
\hline \multirow{5}{*}{6} & 0.8 & 1.12 & 3.53 & 106 & 87 & 121 & 91 & 272 & 322 & 372 & 340 \\
\hline & 0.2 & 0.10 & 2.28 & 12 & 39 & 39 & 39 & 53 & 53 & 57 & 56 \\
\hline & 0.4 & 0.20 & 2.31 & 17 & 36 & 39 & 35 & 52 & 55 & 61 & 58 \\
\hline & 0.6 & 0.30 & 2.37 & 23 & 33 & 40 & 32 & 51 & 57 & 69 & 61 \\
\hline & 0.8 & 0.43 & 2.46 & 30 & 29 & 42 & 30 & 52 & 60 & 78 & 65 \\
\hline
\end{tabular}

only focus on the exchange-correlation component $\sigma_{\mathrm{xc}}$ of the total surface energy. The surface exchange-correlation energy is

$$
\sigma_{\mathrm{xc}}=\int_{-\infty}^{\infty} d x n(x)\left[\epsilon_{\mathrm{xc}}(x)-\epsilon_{\mathrm{xc}}(-\infty)\right],
$$

and may be decomposed as a sum of the exchange and correlation contributions $\sigma_{\mathrm{xc}}=\sigma_{\mathrm{x}}+\sigma_{\mathrm{c}}$

We evaluated the surface exchange and correlation energies of spin-polarized jellium produced by the two model external magnetic fields of Eqs. (31) and (32). The results are displayed in Table I. The surface exchange and correlation energies of the spin-unpolarized jellium are also listed for comparison. In our calculations, we employed LSD orbitals and densities obtained by self-consistently solving the KohnSham equation (27) using the LSD exchange-correlation po- tential. For a justification of this approach, see Ref. 47. The work functions shown in Table I are the LSD values.

Previous studies ${ }^{11}$ show that, like PKZB, TPSS successfully improves upon LSD in the surface exchange-correlation energy of a spin-unpolarized jellium, while PBE gives a correction of the wrong sign to LSD and thus underestimates this quantity. Figures 1 and 2 clearly show that, while the surface exchange energy of LSD is much more overestimated than those of the PBE GGA and TPSS meta-GGA, the surface exchange-correlation energies of these density functionals are not very different from each other. Figure 2 shows that the improvement of TPSS over LSD and PBE may be attributed to its recovery of the known correct gradient expansions of the exchange (Refs. 23 and 24)-correlation ${ }^{48-50}$ energy. Pictured in Fig. 2 is the dependence of the exchangecorrelation enhancement factor, 


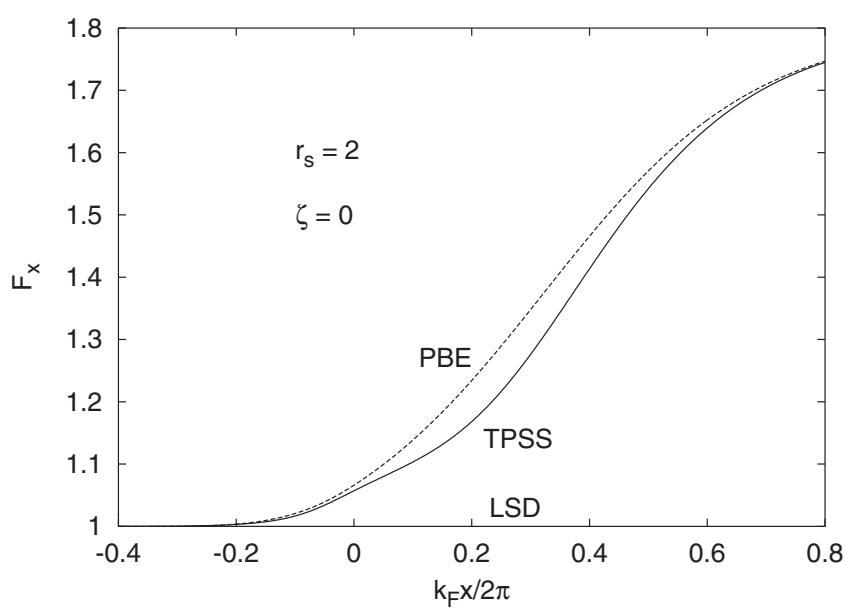

FIG. 1. Exchange enhancement factor $F_{\mathrm{x}}$ of Eq. (5) for LSD, PBE, and TPSS as functions of position $x$ in units of $2 \pi / k_{F}$ relative to the jellium edge $(x=0)$ for spin-unpolarized jellium with $r_{s}=2$.

$$
F_{\mathrm{xc}}=\epsilon_{\mathrm{xc}}^{\mathrm{TPSS}}\left(n_{\uparrow}, n_{\downarrow}, \nabla n_{\uparrow}, \nabla n_{\downarrow}, \tau_{\uparrow}, \tau_{\downarrow}\right) / \epsilon_{\mathrm{x}}^{\mathrm{unif}}(n),
$$

upon position $x$.

Table I suggests that the work function and the surface exchange-correlation energy of a metal could be slightly reduced by an increase in bulk spin polarization due to ferromagnetism $\left[B=B_{0} \theta(-x)\right]$ but could be strongly increased by an increase in spin polarization due to an external uniform magnetic field. For the work function of the model ferromagnet, our results agree qualitatively with those of Kautz and Schwartz ${ }^{9}$ [who, however, used random phase approximation (RPA) input to their LSD calculation].

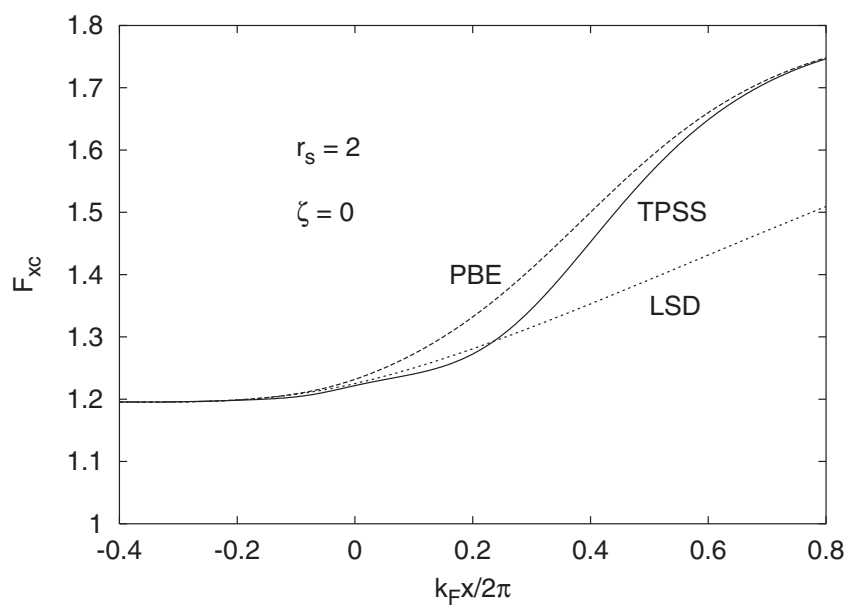

FIG. 2. Exchange-correlation enhancement factors $F_{\mathrm{xc}}$ of Eq. (36) for LSD, PBE, and TPSS as functions of position $x$ in units of $2 \pi / k_{F}$ relative to the jellium edge $(x=0)$ for spin-unpolarized jellium with $r_{s}=2$. The same (LSD) surface density profile $n(x)$ is assumed for all three functionals. Thus, the differences in $F_{\mathrm{xc}}(x)$ shown here determine the differences in surface exchangecorrelation energy $\sigma_{\mathrm{xc}}$ seen in the $\zeta=0$ row of Table I. The higher is $F_{\mathrm{xc}}$ at a given $x$, the lower is $\sigma_{\mathrm{xc}}$. However, the large- $x$ tail region of low density is much less important than the region close to the edge of the positive background at $x=0$.

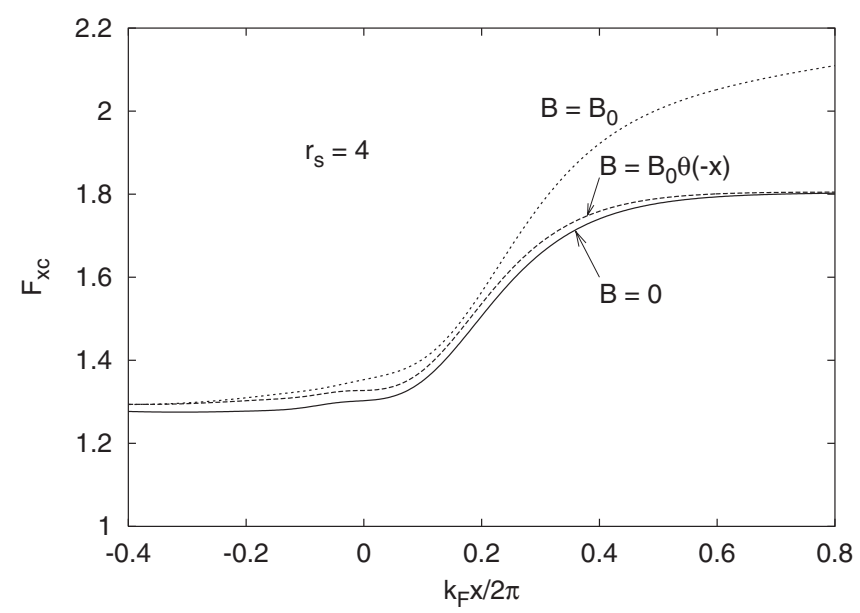

FIG. 3. Comparison of TPSS exchange-correlation enhancement factor $F_{\mathrm{xc}}$ of Eq. (36) as a function of $x$ in units of $2 \pi / k_{F}$ relative to the jellium edge $(x=0)$ for spin-unpolarized jellium at $r_{s}=4$ and for spin-polarized jellium at $r_{s}=4$ and $\zeta=0.4$ produced by the two external magnetic fields of Eqs. (31) and (32).

The local spin polarization $\zeta(x)$ varies from its bulk value at $x=-\infty$ to a limiting tail value $\zeta(\infty)$ at $x=+\infty$. For $B$ $=B_{0} \theta(-x), \zeta(\infty)=0$, but for $B=B_{0}, \zeta(\infty)=1$. These different limits are reflected in Fig. 3, a plot of exchange-correlation enhancement factors vs position $x$, and are presented directly in Fig. 4.

\section{B. Spin dependence of PBE GGA and TPSS meta-GGA}

To examine the spin dependences of the PBE GGA and TPSS meta-GGA for a spin-polarized jellium, we define the surface exchange-correlation spin-polarization energy as

$$
\Delta \sigma_{\mathrm{xc}}=\sigma_{\mathrm{xc}}\left(r_{s}, \zeta\right)-\sigma_{\mathrm{xc}}\left(r_{s}, 0\right) .
$$

This quantity $\Delta \sigma_{\mathrm{xc}}$ can tell us how the surface exchangecorrelation energy of a spin-polarized jellium changes when

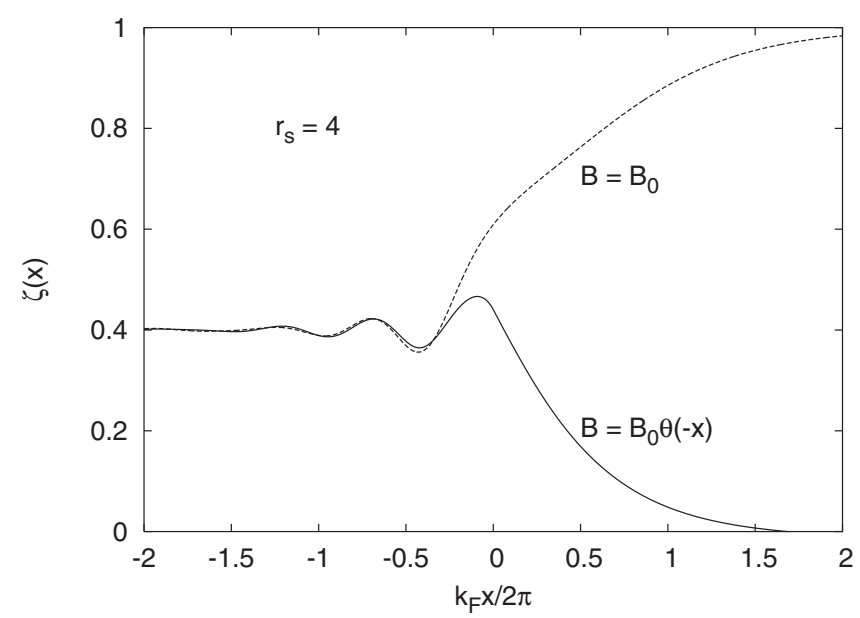

FIG. 4. Relative spin polarization $\zeta=\left(n_{\uparrow}-n_{\downarrow}\right) / n$ as a function of $x$ in units of $2 \pi / k_{F}$ relative to the jellium edge $(x=0)$ for spinpolarized jellium at $r_{s}=4$ and $\zeta=0.4$ produced by two external magnetic fields of Eqs. (31) and (32). 
TABLE II. Spin dependences of the surface exchange-correlation energies (in units of $\mathrm{erg} / \mathrm{cm}^{2}$ ) of the planar jellium surface in PBE and TPSS with various bulk valence densities. LSD orbitals and densities are used.

\begin{tabular}{|c|c|c|c|c|c|c|}
\hline \multirow[b]{2}{*}{$r_{s}$} & \multirow[b]{2}{*}{$\sigma_{\mathrm{xc}}^{\mathrm{TPSS}}\left(r_{s}, 0\right)$} & & \multicolumn{4}{|c|}{$\left[\sigma_{\mathrm{xc}}\left(r_{s}, \zeta\right)-\sigma_{\mathrm{xc}}\left(r_{s}, 0\right)\right]$} \\
\hline & & & 0.2 & 0.4 & 0.6 & 0.8 \\
\hline \multicolumn{7}{|c|}{$B(\mathbf{r})=B_{0} \theta(-x)$} \\
\hline \multirow[t]{2}{*}{2} & 3380 & PBE & 0 & -2 & -18 & -83 \\
\hline & & TPSS & 0 & -3 & -20 & -86 \\
\hline \multirow[t]{2}{*}{4} & 266 & PBE & -1 & -2 & -4 & -12 \\
\hline & & TPSS & -1 & -2 & -6 & -12 \\
\hline \multirow[t]{2}{*}{6} & 55 & PBE & -1 & -1 & -3 & -5 \\
\hline & & TPSS & 0 & -1 & -3 & -4 \\
\hline \multicolumn{7}{|c|}{$B(\mathbf{r})=B_{0}$} \\
\hline \multirow[t]{2}{*}{2} & 3380 & PBE & 205 & 619 & 1060 & 1441 \\
\hline & & TPSS & 206 & 621 & 1074 & 1473 \\
\hline \multirow[t]{2}{*}{4} & 266 & PBE & 7 & 24 & 46 & 69 \\
\hline & & TPSS & 7 & 23 & 46 & 74 \\
\hline \multirow[t]{2}{*}{6} & 55 & PBE & 1 & 3 & 5 & 8 \\
\hline & & TPSS & 1 & 3 & 6 & 10 \\
\hline
\end{tabular}

$\zeta$ changes. Tables II-IV show the comparison of the spin dependences of PBE and TPSS for normal bulk valence densities and when the enhancement factor $F_{\mathrm{xc}}$ of Eq. (36) is uniformly scaled to the high-density $\left(r_{s} \rightarrow 0\right.$ or exchangeonly) and low-density ${ }^{27,33}$ ( $r_{s} \rightarrow \infty$ or strong-interaction) limits, respectively. From Tables II-IV, we see that TPSS has nearly the same dependence as PBE in every case we examined here, suggesting that our earlier procedure to construct the spin-dependence of $C(\zeta, \xi)$ in Eq. (13) is right.

The PBE GGA and TPSS meta-GGA describe magnetism very similarly at jellium surfaces. Whether this will remain true for real systems remains to be determined. We only know of a study of the ground-state spins of iron complexes, ${ }^{51}$ which compares PBE with TPSS hybrid ${ }^{19}$ (a hybrid of TPSS with $10 \%$ exact exchange), and another study of iron complexes, ${ }^{52}$ which seems to show similar energy gaps between high- and low-spin states from PBE and TPSS. A private communication ${ }^{53}$ from one of the authors of Ref. 51 shows that PBE and TPSS give similar energy differences among three spin states in each of seven iron complexes.

TABLE III. Spin dependences of the surface exchange-correlation energies of the planar jellium surface in PBE and TPSS, when the enhancement factor $F_{\mathrm{xc}}$ of Eq. (36) is uniformly scaled to the high-density $\left(r_{s} \rightarrow 0\right)$ or exchange-only limit from normal bulk valence densities. LSD orbitals and densities are used $\left(\mathrm{erg} / \mathrm{cm}^{2}\right)$.

\begin{tabular}{|c|c|c|c|c|c|c|}
\hline \multirow{2}{*}{\multicolumn{2}{|c|}{$\begin{array}{c}\sigma_{\mathrm{x}}^{\mathrm{TPSS}}\left(r_{s}, 0\right) \\
\left(\mathrm{erg} / \mathrm{cm}^{2}\right)\end{array}$}} & & \multicolumn{4}{|c|}{$\left[\sigma_{\mathrm{x}}\left(r_{s}, \zeta\right)-\sigma_{\mathrm{x}}\left(r_{s}, 0\right)\right]$} \\
\hline & & & 0.2 & 0.4 & 0.6 & 0.8 \\
\hline \multicolumn{7}{|c|}{$B(\mathbf{r})=B_{0} \theta(-x)$} \\
\hline \multirow[t]{2}{*}{2} & 2553 & PBE & 5 & 15 & 25 & 14 \\
\hline & & TPSS & 5 & 16 & 26 & 16 \\
\hline \multirow[t]{2}{*}{4} & 141 & PBE & 1 & 4 & 9 & 13 \\
\hline & & TPSS & 2 & 5 & 9 & 15 \\
\hline \multirow[t]{2}{*}{6} & 15 & PBE & 0 & 1 & 2 & 3 \\
\hline & & TPSS & 1 & 2 & 3 & 4 \\
\hline \multicolumn{7}{|c|}{$B(\mathbf{r})=B_{0}$} \\
\hline \multirow[t]{2}{*}{2} & 2553 & PBE & 250 & 740 & 1261 & 1704 \\
\hline & & TPSS & 250 & 742 & 1265 & 1709 \\
\hline \multirow[t]{2}{*}{4} & 141 & PBE & 12 & 39 & 73 & 107 \\
\hline & & TPSS & 13 & 40 & 74 & 108 \\
\hline \multirow[t]{2}{*}{6} & 15 & PBE & 2 & 7 & 13 & 19 \\
\hline & & TPSS & 3 & 7 & 14 & 15 \\
\hline
\end{tabular}


TABLE IV. Spin dependences of the surface exchange-correlation energies of the planar jellium surface in PBE and TPSS when the enhancement factor $F_{\mathrm{xc}}$ of Eq. (36) is uniformly scaled to the low-density $\left(r_{s}\right.$ $\rightarrow \infty$ ) limit from normal bulk valence densities. LSD orbitals and densities are used. The $\zeta$ dependence here arises not so much from $F_{\mathrm{xc}}$ as from the $\zeta$ dependence of the surface density profile $n(x)$, which for $B(\mathbf{r})$ $=B_{0}$ spreads out more as $|\zeta|$ increases $\left(\mathrm{erg} / \mathrm{cm}^{2}\right)$.

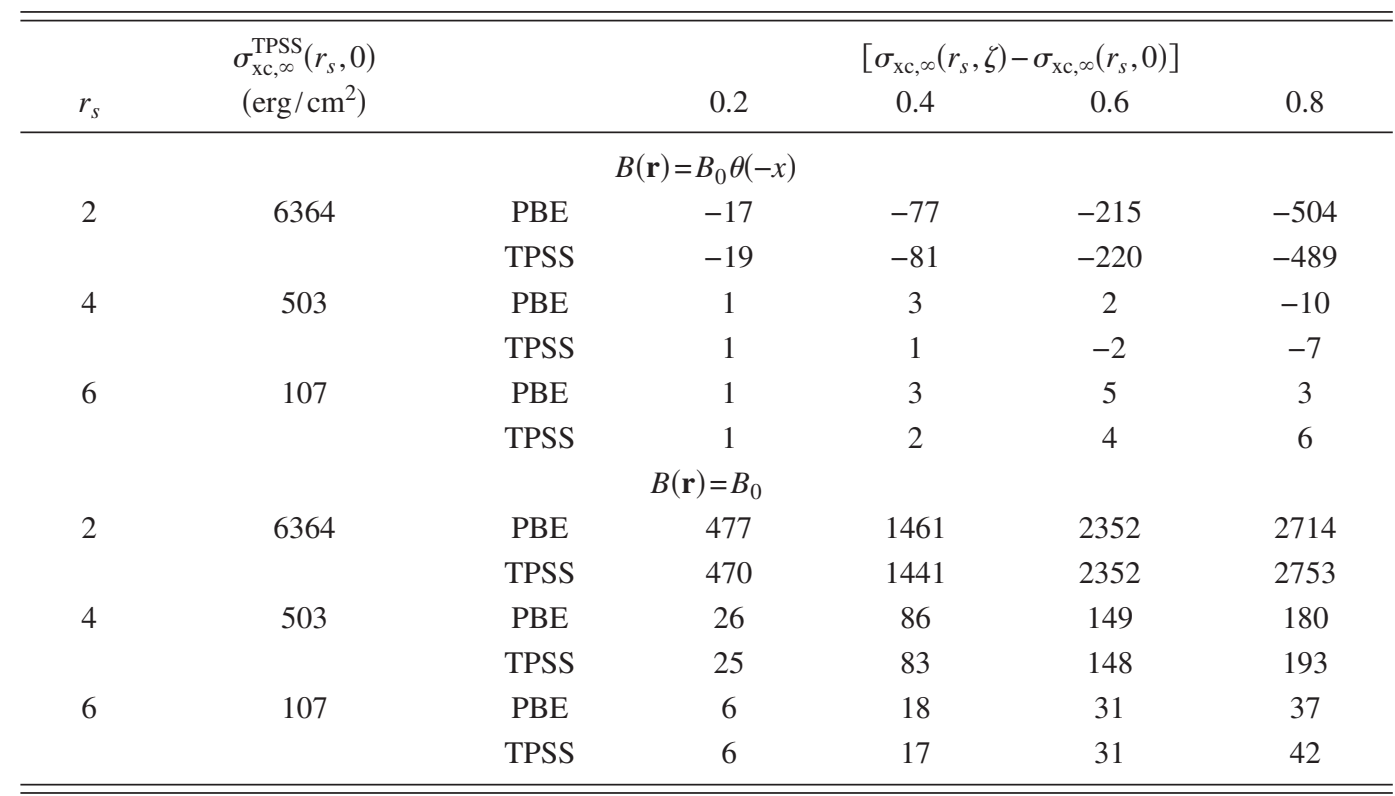

\section{INFINITE BARRIER MODEL OF THE JELLIUM SURFACE}

The earliest surface model of a metal is the infinite barrier model proposed by Bardeen, ${ }^{54}$ in which the surface density profile is that of noninteracting free electrons in the presence of a hard wall. This is an oversimplified surface model of a jellium metal because many properties of this model surface cannot be transferred to real metal surfaces. However, it may serve as an ideal model system with rapidly varying densities and can be employed to test density functionals because the exact electron density, the kinetic energy density, and the conventional exchange energy per electron of this model system are analytically known. ${ }^{34,55}$ Here, the surface exchangecorrelation energies of the LSD, PBE, PKZB, and TPSS are evaluated for normal bulk valence densities, and when the enhancement factor $F_{\text {xc }}$ of Eq. (36) is uniformly scaled to the high-density or exchange-only and low-density or stronginteraction limits. The results are shown in Table V.

$\mathrm{RPA}+$ is a sophisticated approximation involving the full RPA plus a GGA for the short-range correction to RPA. The
$\mathrm{RPA}+$ values in Table $\mathrm{V}$ are evaluated from ${ }^{56}$

$$
\sigma_{\mathrm{xc}}^{\mathrm{RPA}+}=0.0714\left(1+3.451 r_{s}\right) /\left(1+1.688 r_{s}\right),
$$

which is a fit to the RPA+ values of Yan et al. ${ }^{57}$ at $r_{s}=0$, 2.07, 4, and 6. The RPA+ values are exact in the exchangeonly or $r_{s} \rightarrow 0$ limit, and we take them to be nearly exact for all $r_{s}$. We see from Table $\mathrm{V}$ that the order of accuracy of these functionals for normal bulk valence densities is $\sigma_{\mathrm{xc}}^{\mathrm{PBE}}$ $<\sigma_{\mathrm{xc}}^{\mathrm{PKZB}} \approx \sigma_{\mathrm{xc}}^{\mathrm{TPSS}}<\sigma_{\mathrm{xc}}^{\mathrm{LSD}}$, while, in the high-density or exchange-only limit, we have $\sigma_{\mathrm{x}}^{\mathrm{LSD}}<\sigma_{\mathrm{x}}^{\mathrm{PBE}}<\sigma_{\mathrm{x}}^{\mathrm{PKZB}} \approx \sigma_{\mathrm{x}}^{\mathrm{TPSS}}$.

\section{ENERGIES OF JELLIUM SPHERES}

In the spherical jellium model, a positive charge background is contained inside a sphere of radius

$$
R=r_{s} N^{1 / 3}
$$

The potential due to the positive background charge is

TABLE V. Surface exchange-correlation energy of jellium in the infinite barrier model. March's unit $3 e^{2} \bar{n} / 4 \pi=\left(88738 / r_{s}^{3}\right)\left(\mathrm{erg} / \mathrm{cm}^{2}\right)$ is used (Ref. 34). The RPA+ value for $r_{s}=0$ is from Ref. 58 .

\begin{tabular}{cccccc}
\hline \hline$r_{s}$ & RPA + & LSD & PBE & PKZB & TPSS \\
\hline 0 & 0.0714 & 0.1108 & 0.0452 & 0.0501 & 0.0516 \\
2 & 0.1289 & 0.1222 & 0.1035 & 0.1079 & 0.1098 \\
4 & 0.1364 & 0.1296 & 0.1190 & 0.1235 & 0.1255 \\
6 & 0.1393 & 0.1350 & 0.1286 & 0.1335 & 0.1354 \\
$\infty$ & & 0.2157 & 0.2313 & 0.2588 & 0.2603 \\
\hline \hline
\end{tabular}




$$
v_{+}(r)= \begin{cases}-\frac{N}{2 R}\left[3-\left(\frac{r}{R}\right)^{2}\right] & (r \leq R) \\ -\frac{N}{r} & (r>R) .\end{cases}
$$

The calculation of the exchange and correlation energies was done in an a posteriori process using LSD densities as input, as explained in Ref. 47. By solving the many-electron problem using the Kohn-Sham approach, we may obtain a series of single-particle levels. For metallic densities, the energy ordering of these states is $1 s, 1 p, 1 d, 2 s, 1 f, 2 p, 1 g, 2 d, 1 h$, $3 s, 2 f, \ldots$ The filling of shells yields special stability for the so-called magic clusters.

As in Ref. 47, we calculated the energies of jellium spheres for some magic numbers $(N=2,8,18,20,34,40,58$, 92 , and 106) with various densities $r_{s}=2.07,3.25,4.00$, and 5.62. We chose these magic numbers and densities due to the availability of the respective results in diffusion quantum Monte Carlo (DQMC) calculations by Sottile and Ballone S9 $^{59}$ with which we want to compare. These DQMC results on jellium spheres are supposed to be the most accurate available for the systems under study. However, the SottileBallone values ${ }^{59}$ are affected by a systematic error in the correlation energies due to their fixed-node assumption.

For $N \rightarrow \infty$, we get the limit of the uniform electron gas for which the fixed-node error may be estimated as the difference between the fixed-node calculation done by Ortiz and Ballone ${ }^{60}$ and the released-node calculation of Ceperley and Alder. ${ }^{61}$ This error estimate for the uniform electron gas has been presented in Table 7 of Ref. 47. For a sphere with $N=2$ electrons, there is no node in the space factor of the ground-state wave function, so the fixed-node error is absent. Using the limits $N=\infty$ and $N=2$, we may interpolate the fixed-node error of the intermediate spheres by multiplying the correction to the correlation energy in the uniform electron gas by a factor suggested by the liquid drop model $(\mathrm{LDM})^{62}$ [Eq. (41))],

$$
\Delta \epsilon_{\mathrm{c}}(N)=\Delta \epsilon_{\mathrm{c}}^{\text {unif }}\left[1-\left(\frac{2}{N}\right)^{1 / 3}\right] .
$$

The uniform electron gas correction $\Delta \epsilon_{\mathrm{c}}^{\mathrm{unif}}$ is equal to the $\Delta \epsilon_{\mathrm{c}}^{O B}$ of Table 7 of Ref. 47.

A better comparison of the energetics produced by the various density functionals can now be done. The effect of correction is shown in Fig. 5 for a single density $\left(r_{s}\right.$ $=4.0$ bohr). The error of a density functional is the difference between the exchange-correlation energy and the corresponding corrected DQMC value. Table VI shows the errors of the different density functionals for the total energy per electron, and for the five indicated densities, averaged over the magic closed-shell clusters in the range $2 \leq N \leq 106$. A supplementary table showing deviations from corrected fixed-node DQMC values [Ref. 59 and Eq. (40)] of the total energies per electron for individual jellium clusters is available through the EPAPS depository. ${ }^{63}$

Table VII displays the relative errors in the correlation energies, again averaged over the closed-shell spheres. The improvement of all functionals with respect to LSD is clear

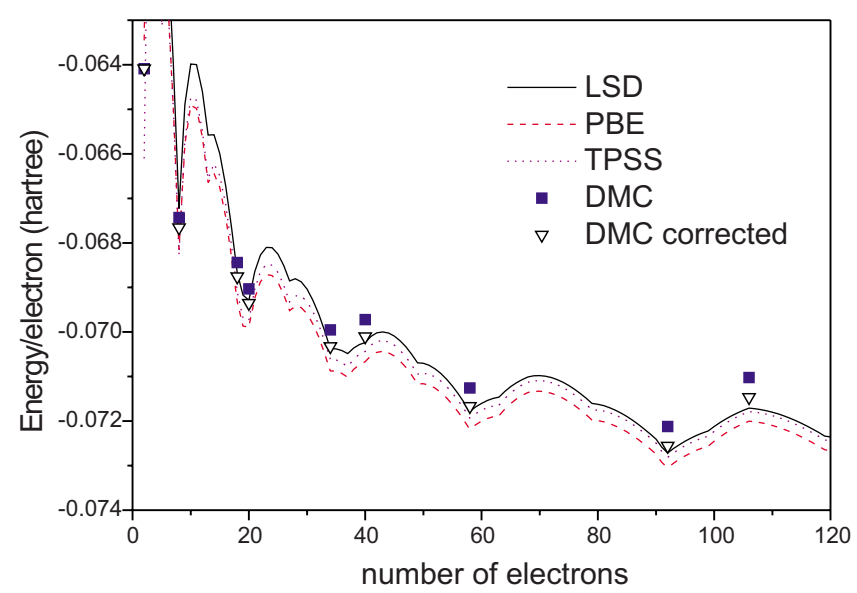

FIG. 5. (Color online) Total energies per electron of jellium spheres for $r_{s}=4$. The effect of our fixed-node correction is visible for magic clusters $N=2,8,18,20,34,40,58,92$, and 106. PBE is too low, but TPSS and especially LSD are good for $N>2$. LSD is best for $N>2$ but worst for $N=2$. Although TPSS surface energies are slightly higher than LSD values, TPSS curvature energies (Fig. 6 and Table IX) are lower, leading to a slightly lower TPSS total energy for these spheres. All curves tend as $N \rightarrow \infty$ to -0.0774 hartree.

and the TPSS shows the smallest deviation in correlation energy.

Surface and curvature energies are relevant ${ }^{62}$ not only to clusters and voids but even to cohesive energies and monovacancy formation energies. Adopting the same fitting procedure for extracting $\mathrm{LDM}^{62}$ parameters from jellium spheres as in Ref. 47, we use the following equation for the energy of a neutral jellium cluster with $N$ valence electrons:

$$
\frac{E^{\mathrm{LDM}}}{N}=\epsilon^{\mathrm{unif}}+4 \pi r_{s}^{2} \sigma N^{-1 / 3}+2 \pi r_{s} \gamma N^{-2 / 3},
$$

where $\sigma$ and $\gamma$ describe the surface and curvature energies, respectively, and $\epsilon^{\mathrm{unif}}=\left(4 \pi r_{s}^{3} / 3\right) \alpha$ is the energy per electron of the uniform electron gas, with $\alpha$ being its energy per volume. This model neglects the quantum oscillations in the energy due to the shell structure. For the sequence of closed-

TABLE VI. Mean absolute deviations from corrected fixed-node DQMC values [Ref. 59 and Eq. (40)] of the total energies per electron of jellium spheres in various density functional approaches. The values are averages over the magic clusters $N=2,8,18,20,34$, 40, 58, 92, and 106. For individual $N$ at $r_{s}=4$, see Fig. 5.

\begin{tabular}{ccccc}
\hline \hline & \multicolumn{4}{c}{$\left|\left(E-E^{\mathrm{DQMC}}\right) / N\right|($ hartree $)$} \\
$r_{s}$ & LSD & PBE & PKZB & TPSS \\
\hline 1.00 & 0.0040 & 0.0015 & 0.0015 & 0.0007 \\
2.00 & 0.0018 & 0.0007 & 0.0004 & 0.0004 \\
3.25 & 0.0007 & 0.0005 & 0.0002 & 0.0004 \\
4.00 & 0.0004 & 0.0005 & 0.0004 & 0.0005 \\
5.62 & 0.0005 & 0.0006 & 0.0006 & 0.0007 \\
Average & 0.0015 & 0.0008 & 0.0006 & 0.0006 \\
\hline \hline
\end{tabular}


TABLE VII. Average relative deviations of the correlation energy of jellium spheres, in various density functional approaches, from corrected DQMC values [Ref. 59 and Eq. (40)]. Averages were taken over magic clusters: $N=2,8,18,20,34,40,58,92$, and 106.

\begin{tabular}{ccccc}
\hline \hline \multicolumn{5}{c}{$\left(E_{\mathrm{c}}-E_{\mathrm{c}}^{\mathrm{DQMC}}\right) / E_{\mathrm{c}}^{\mathrm{DQMC}}$} \\
$r_{s}$ & LSD & PBE & PKZB & TPSS \\
& $(\%)$ & $(\%)$ & $(\%)$ & $(\%)$ \\
\hline 1.00 & 40.3 & 6.7 & 7.4 & 5.9 \\
2.00 & 34.0 & 7.7 & 7.8 & 6.5 \\
3.25 & 29.7 & 7.3 & 6.9 & 5.8 \\
4.00 & 27.2 & 6.4 & 5.9 & 4.9 \\
5.62 & 26.4 & 7.3 & 6.4 & 5.5 \\
Average & 31.5 & 7.1 & 6.9 & 5.7 \\
\hline \hline
\end{tabular}

shell clusters, the oscillation is presumably the same in LSD as at any higher level of theory, so the difference cancels out. (More generally, $\left[E-E^{\mathrm{LSD}}\right] / N$ in finite systems can be extrapolated smoothly to infinite size. ${ }^{64}$ ) Thus, the LDM equation for the closed-shell clusters, including the smaller ones, is better written as the difference to $\mathrm{LSD},{ }^{47}$

$$
\begin{aligned}
\frac{E}{N}-\frac{E^{\mathrm{LSD}}}{N}= & \left(\epsilon^{\mathrm{unif}}-\epsilon_{\mathrm{PW} 92}^{\mathrm{unif}}\right)+4 \pi r_{s}^{2}\left(\sigma-\sigma^{\mathrm{LSD}}\right) N^{-1 / 3} \\
& +2 \pi r_{s}\left(\gamma-\gamma^{\mathrm{LSD}}\right) N^{-2 / 3}
\end{aligned}
$$

As the functionals PBE, PKZB, and TPSS have the same parametrization (PW92) ${ }^{43}$ in the limit of the uniform electron gas, the first term in the right-hand side is zero.

We used surface energies calculated by a planar surface code of Monnier and Perdew ${ }^{8}$ and reported in Table VIII. Thus, only the curvature-energy term in Eq. (42) needs to be fitted. To perform this fit, we extended the calculation of jellium spheres up to $N=1100$ (except in the case of $r_{s}$ $=5.62$, where we only reached up to $N=748$ ). We checked the "Aufbau" principle, i.e., the highest occupied KohnSham orbital should have energy lower than the lowest unnoccupied orbital, and we only took the clusters obeying that principle. An example of such fitting is plotted in Fig. 6.

Using the resulting fit to curvature-energy differences and the LSD curvature energies given by Ziesche et al., ${ }^{65}$ we show in Table IX our curvature energies for several densities. The energies are, in fact, very similar to a previous

TABLE VIII. Surface exchange-correlation energies of jellium calculated with a planar surface code. Energies are in $\mathrm{erg} / \mathrm{cm}^{2}$. Only deviations from LSD are relevant to fitting Eq. (42).

\begin{tabular}{ccccc}
\hline \hline$r_{s}$ & LSD & PBE & PKZB & TPSS \\
\hline 2.07 & 2961 & 2881 & 3002 & 2985 \\
2.65 & 1204 & 1167 & 1221 & 1215 \\
3.24 & 575.1 & 555.9 & 583.1 & 581.8 \\
3.93 & 279.8 & 269.9 & 283.8 & 284.0 \\
5.62 & 69.89 & 67.27 & 71.15 & 71.90 \\
\hline \hline
\end{tabular}
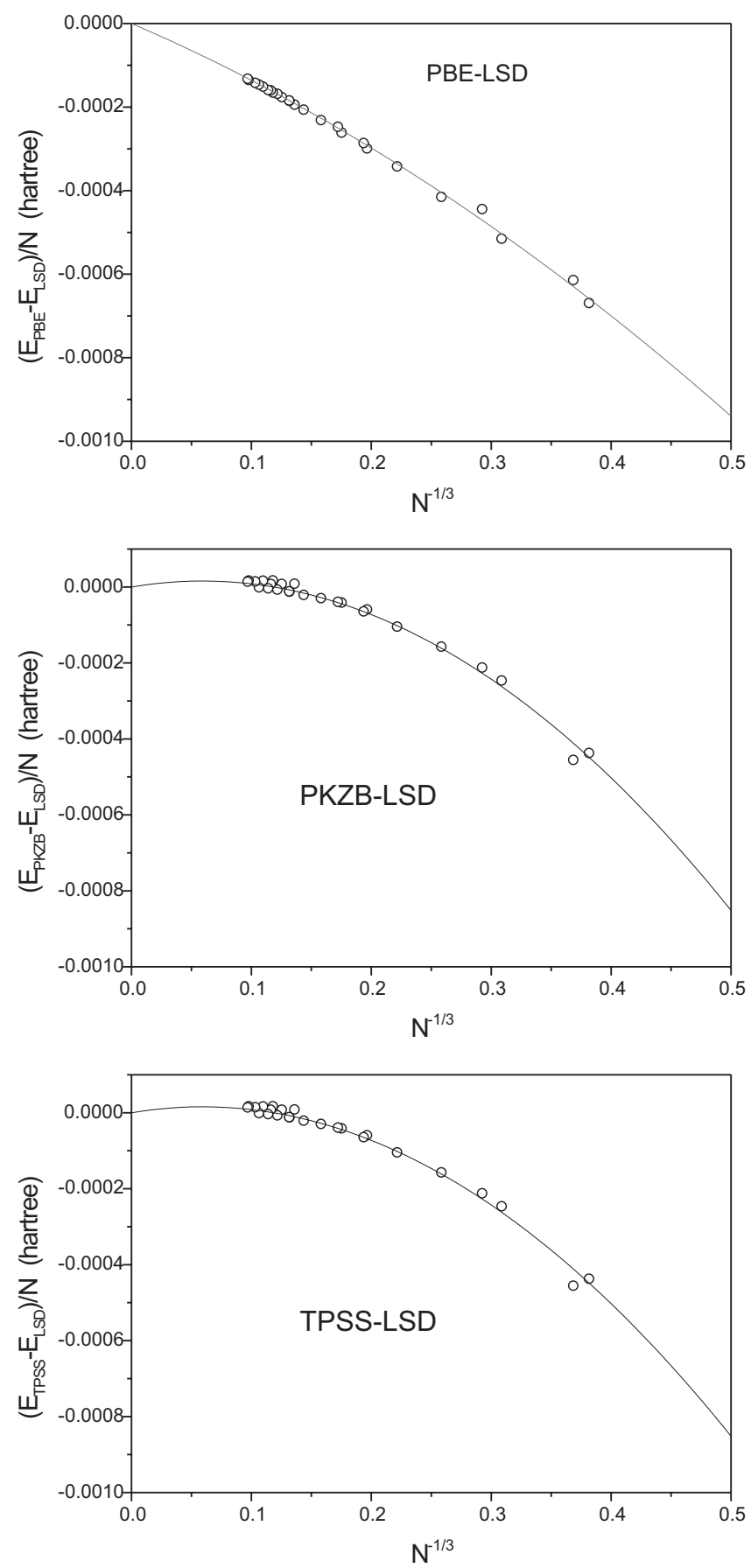

FIG. 6. Deviation from LSD of PBE, PKZB, and TPSS energies for jellium spheres with $r_{s}=3.93$. The full lines are parabolas fitted to the LDM via Eq. (42), as explained in the text. The open circles are input values for the magic clusters respecting the Aufbau principle. The second derivative of this curve at $N^{-1 / 3}=0$ is proportional to the difference of curvature energies between the given functional and LSD.

calculation, ${ }^{47}$ which used only a smaller number of spheres and did not restrict the surface energy term. The curvature energies of TPSS are close to those of PKZB, as expected. The PKZB curvature energies are smaller than those of LSD and PBE, as predicted in Ref. 47. 
TABLE IX. Curvature total energies of jellium (in units of mhartree/bohr) of jellium. Only deviations from LSD are relevant to fitting [Eq. (42)].

\begin{tabular}{ccccc}
\hline \hline$r_{s}$ & LSD & PBE & PKZB & TPSS \\
\hline 2.07 & 1.830 & 1.494 & 0.988 & 1.063 \\
2.65 & 1.044 & 0.885 & 0.548 & 0.609 \\
3.24 & 0.635 & 0.546 & 0.312 & 0.358 \\
3.93 & 0.369 & 0.318 & 0.156 & 0.189 \\
5.62 & 0.180 & 0.161 & 0.082 & 0.097 \\
\hline \hline
\end{tabular}

\section{LINEAR DENSITY RESPONSE AND CHARGE DENSITY WAVES}

There are several reasons why the linear response of the homogenous electron gas to an external perturbation is of interest. First, it is an important step toward a qualitative understanding of the electronic structure of the simple metals, via pseudopotential perturbation theory. ${ }^{66}$ Second, comparing the response obtained from approximate density functionals to the one obtained from quantum Monte Carlo calculations can serve as a test of different density functionals. Third, the linear response relations can be used to study the instability of the uniform phase of jellium against the formation of a charge-density wave. ${ }^{67}$

In the present work, we calculate the linear response for LSD, ${ }^{43}$ PBE GGA, ${ }^{18}$ and the PKZB $^{22}$ and TPSS $^{23}$ metaGGAs. The quantity of interest is the response function $\gamma_{\mathrm{xc}}(\mathbf{q})$ defined by the linear response relation

$$
\delta v_{\mathrm{xc}}(\mathbf{k})=-\frac{\pi}{k_{F}^{2}} \gamma_{x c}\left(\frac{k}{2 k_{F}}\right) \delta n(\mathbf{k}) .
$$

This equation relates the Fourier component $\delta n(\mathbf{k})$ of a density perturbation to the Fourier component $\delta v_{\mathrm{xc}}(\mathbf{k})$ of the exchange-correlation potential that results from the perturbed density. We calculated $\gamma_{\mathrm{xc}}$ separately for the exchange and correlation parts of the different functionals by inserting the perturbed density $n(\mathbf{r})=n_{0}+n_{\mathbf{k}} \cos (\mathbf{k r})$, where $n_{0}=k_{F}^{3} /\left(3 \pi^{2}\right)$ and $n_{\mathbf{k}} \ll n_{0}$, into the energy functional. The resulting expression can be rewritten as a power series in $n_{\mathbf{k}} / n_{0}$ and $\gamma$ is obtained by multiplying the second-order coefficient of this expansion by $-\frac{2 k_{F}^{2}}{\pi}$. (The factor 2 takes care of the $1 / 2$ in the Taylor expansion.) This procedure straightforwardly yields $\gamma_{\mathrm{x}}^{\mathrm{LSD}}=1$ and $\gamma_{\mathrm{x}}^{\mathrm{PBE}}=1+\frac{9}{2} \mu y^{2}$, where $y=\frac{k}{2 k_{F}}$ and $\mu=\beta \pi^{2 / 3}$, with $\beta=0.066725$ being the coefficient of the second-order gradient term in the gradient expansion of the correlation energy in the high-density limit. ${ }^{48}$

For the meta-GGA functionals, the situation is more complicated because they also depend on the kinetic energy density $\tau$, which must be calculated from the orbitals. We can, however, obtain the linear response without knowing the orbitals in certain limiting cases by using the appropriate gradient expansions of the kinetic energy density. For a slowly varying perturbation, i.e., $k \rightarrow 0$, the gradient expansion reads 68

$$
\tau \approx \frac{3}{10}\left(3 \pi^{2}\right)^{2 / 3} n^{5 / 3}+\frac{1}{6} \nabla^{2} n+\frac{1}{360} \frac{\nabla^{4} n}{\left(3 \pi^{2}\right)^{2 / 3} n^{2 / 3}} .
$$

We here retained only terms up to order $n_{\mathbf{k}}$ since higher orders will not contribute to the linear response. Inserting this expression into the TPSS energy functional, Eq. (3) of Ref. 23, and again expanding into a series in $n_{\mathbf{k}} / n_{0}$, we obtain after some algebra the slowly varying limit of the TPSS linear response function,

$$
\gamma_{\mathrm{x}, \text { slow }}^{\text {TPS }}=1+\frac{5}{9} y^{2}+\frac{73}{225} y^{4}-\frac{146}{3375} y^{6}+\mathcal{O}\left(y^{8}\right) .
$$

In the rapidly varying limit, i.e., $\mathbf{k} \rightarrow \infty$ and $n_{\mathbf{k}} \ll 1$, the kinetic energy density can be expanded as ${ }^{69}$

$$
\tau \approx \frac{3}{10}\left(3 \pi^{2}\right)^{2 / 3} n^{5 / 3}+\frac{1}{8} \frac{|\nabla n|^{2}}{n}+c \nabla^{2} n .
$$

To the best of our knowledge, no value for the coefficient $c$ has been given in the past. We argue that $c=0$ for the following reason: It is known that the von Weizsäcker kinetic energy density is a rigorous lower bound for the kinetic energy density,

$$
\tau \geq \frac{1}{8} \frac{|\nabla n|^{2}}{n}
$$

However, for $c \neq 0$ and any nonvanishing amplitude of the perturbation, one can choose a wave vector to make the $\nabla^{2}$ term in Eq. (46) arbitrarily large and negative for certain points in space. Thus, the only way to avoid the violation of the inequality [Eq. (47)] is to simply require $c=0$. Using Eq. (46) with $c=0$ yields the rapidly varying limit of TPSS linear response,

$$
\gamma_{x, \text { rapid }}^{\mathrm{TPSS}}=1+\frac{5}{9} y^{2}
$$

Finally, interpolating between the two limiting cases of Eqs. (45) and (48) with the Padé approximant

$$
\gamma_{x}^{P, \text { TPSS }}=1+\frac{5}{9} y^{2}+\frac{\frac{73}{225} y^{4}}{\left(1+\frac{2}{45} y^{2}\right)^{3}},
$$

we obtain an expression that can be expected to be very close to the exact TPSS response $\gamma_{x}^{\mathrm{TPSS}}$ for all $k$. Going through the same procedure for the PKZB meta-GGA, we confirmed that PKZB and TPSS have the same linear response.

In Fig. 7, we plotted the exchange-only response functions for LSD, PBE GGA, and TPSS meta-GGA, together with the exact-exchange-only response from Ref. 70. The TPSS exchange-only response is extremely close to the exact-exchange-only response up to $y \approx 0.6$, and both $\mathrm{PBE}$ and TPSS provide reasonable approximations up to $y \approx 1$. Only for wave vectors with a magnitude of more than twice the Fermi wave number do differences become pronounced since the semilocal functionals do not recover the abrupt drop of the exact-exchange response beyond $y \approx 1$.

To calculate the correlation contribution to the LSD linear response, we proceed slightly differently and directly evaluate $\gamma_{\mathrm{c}}^{\mathrm{LSD}}=-\left(k_{F}^{2} / \pi\right) \delta^{2} E_{\mathrm{c}}^{\mathrm{LSD}} / \delta n(\mathbf{r}) \delta n\left(\mathbf{r}^{\prime}\right)$ with the Perdew- 


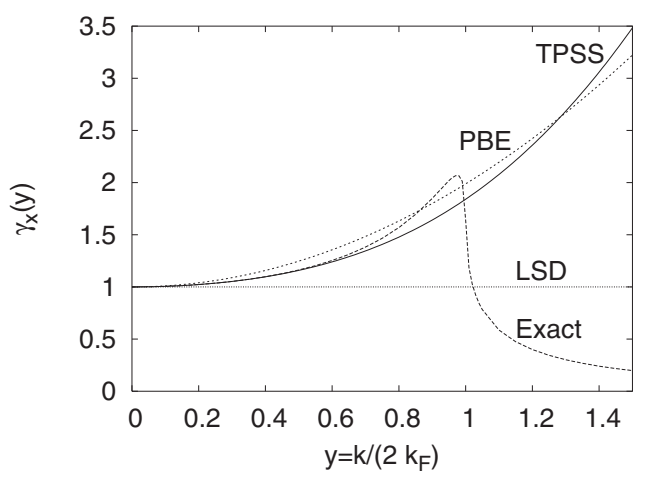

FIG. 7. The exchange-only response function $\gamma_{\mathrm{x}}$ for bulk jellium in the TPSS (full line), PBE (short dashed), and LSD (dotted) functionals. The long dashed line shows the exact-exchange-only results from Ref. 70; see text for discussion.

Wang expression. ${ }^{43} \quad$ Obviously, $\quad \delta^{2} E_{\mathrm{c}}^{\mathrm{LSD}} / \delta n(\mathbf{r}) \delta n\left(\mathbf{r}^{\prime}\right)$ $=\left[2 \partial \epsilon_{\mathrm{c}}^{\text {unif }} / \partial n+n \partial^{2} \epsilon_{\mathrm{c}}^{\text {unif }} / \partial n^{2}\right] \delta\left(\mathbf{r}-\mathbf{r}^{\prime}\right)$, and some algebra yields

$$
\frac{\partial \epsilon_{\mathrm{c}}^{\text {unif }}}{\partial n}=-\frac{4 \pi r_{s}^{4}}{9} A\left[\frac{c_{1} q_{2}}{\sqrt{r_{s} c_{3} q_{1}}}-2 \alpha_{1} \ln \left(1+\frac{1}{c_{2}}\right)\right]
$$

and

$$
\begin{aligned}
\frac{\partial^{2} \epsilon_{\mathrm{c}}^{\text {unif }}}{\partial n^{2}}= & \frac{16}{81} \pi^{2} r_{s}^{7}\left[c_{1} q_{2}^{2}+4 \alpha_{1} q_{1} q_{2} c_{3} \sqrt{r_{s}}-2 c_{1} q_{2}^{2} c_{3}\right. \\
& \left.+c_{1} c_{3} q_{1}\left(-\beta_{1}+3 \beta_{3} r_{s}+8 \beta_{4} r_{s}^{3 / 2}\right) / \sqrt{r_{s}}\right] /\left[8 A q_{1}^{4}\right. \\
& \left.\times\left(1+1 / c_{2}\right)^{2}\right]+4\left[\frac{A c_{1} q_{2}}{\sqrt{r_{s}} q_{1} c_{3}}-2 A \alpha_{1} \ln \left(1+1 / c_{2}\right)\right] .
\end{aligned}
$$

Here, $q_{1}=\beta_{1} \sqrt{r_{s}}+\beta_{2} r_{s}+\beta_{3} r_{s}^{3 / 2}+\beta_{4} r_{s}^{2}, q_{2}=\beta_{1}+2 \beta_{2} \sqrt{r_{s}}+3 \beta_{3} r_{s}$ $+4 \beta_{4} r_{s}^{3 / 2}, c_{1}=1+\alpha_{1} r_{s}, c_{2}=2 A q_{1}$, and $c_{3}=1+c_{2}$, with $A, \alpha_{1}$, and $\beta_{1}-\beta_{4}$ being the parameters from Ref. 43.

The contribution of the correlation part to the response for the PBE GGA and the two meta-GGAs is obtained analogously to the calculations for the exchange functionals. In all three cases, we obtain the same result,

$$
\gamma_{\mathrm{c}}^{\mathrm{PBE}}=\gamma_{\mathrm{c}}^{\mathrm{PKZB}}=\gamma_{\mathrm{c}}^{\mathrm{TPSS}}=\gamma_{\mathrm{c}}^{\mathrm{LSD}}-\frac{3}{2} \beta \pi^{2} y^{2},
$$

where $\beta$ is the same parameter as in the PBE exchange response function (see above).

In Figs. 8 and 9, we compare the response as obtained from the density functionals to the quantum Monte Carlo results of Ref. 71 and the results of Ref. 72 for two different densities. LSD and PBE provide a satisfying average $\gamma$ for $y<1$. The TPSS response shows more structure than LSD and PBE and is also in satisfactory agreement with the quantum Monte Carlo (QMC) results. Only for $y>1.1$ does the TPSS response move outside of the QMC error bars.

Finally, following Ref. 67, we calculated the instability of jellium against the formation of a charge-density wave. The Fourier components of the self-consistent potential are related to the ones of the external potential by

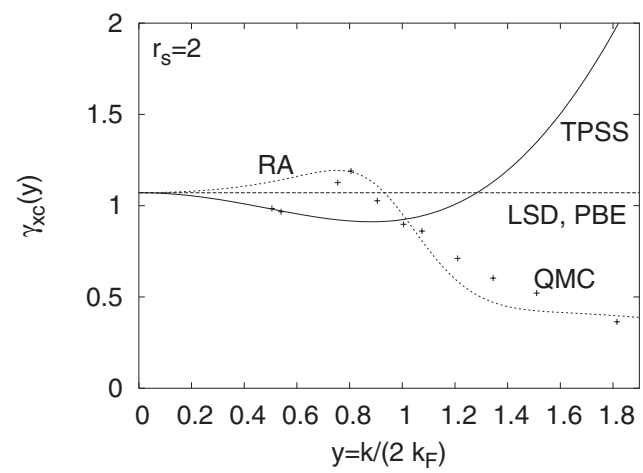

FIG. 8. The exchange-correlation response function $\gamma_{\mathrm{xc}}$ for bulk jellium as obtained from the TPSS functional (full line), the LSD and PBE functionals (long dashed), the Richardson-Ashcroft (RA) approximation (Ref. 72) (short dashed), and the quantum Monte Carlo calculations of Ref. 71 (crosses) for $r_{s}=2$.

$$
v(\mathbf{k})=\frac{1}{1-\frac{4 \pi}{k^{2}}\left[1-y^{2} \gamma_{\mathrm{xc}}(y)\right] \chi(k)} v_{\mathrm{ext}}(\mathbf{k}),
$$

where $\chi$ is the Lindhard response function. For vanishing $v_{\text {ext }}(\mathbf{k})$, the left-hand side of this equation can only be nonzero if the denominator on the right-hand side vanishes. Thus, for each value of $y$, we numerically search for the density (i.e., $k_{F}$ ) that sets the denominator to zero. The largest value of $k_{F}$ (for all $y$ ) found in that way marks the onset of jellium instability. In this way, we confirmed that, for exchange and correlation combined, TPSS does not alter much the prediction of LSD for the onset of jellium instability, which occurs for $k_{F}<0.06$ a.u. $\left(r_{s} \geqslant 30\right)$. However, in TPSS the wave vector for the instability is increased from $1.1\left(2 k_{F}\right)$ to $1.5\left(2 k_{F}\right)$.

\section{CONCLUSIONS}

In conclusion, we have calculated and compared the jellium surface exchange-correlation energies of the PBE GGA and the TPSS meta-GGA and of these two functionals when uniformly scaled to the high- and low-density limits for the normal bulk valence densities in magnetic fields. In all the cases, the fairly good agreement of the PBE GGA with the TPSS meta-GGA shows that the TPSS meta-GGA indeed represents the self-correlation correction of the PBE GGA.

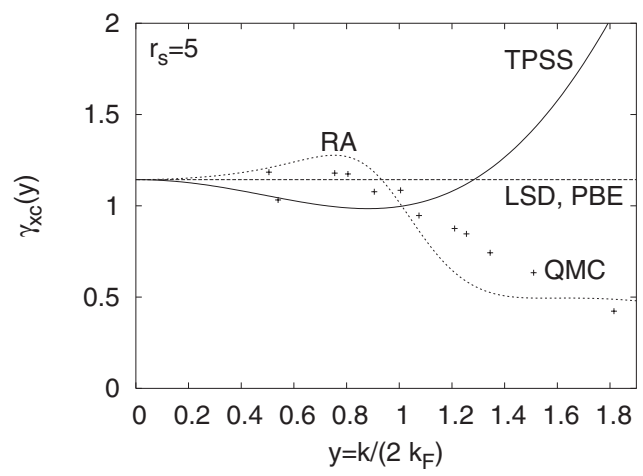

FIG. 9. Same as Fig. 8 but for a density with $r_{s}=5$. 
We have further found that the internal magnetic field of Eq. (31) and the external uniform field of Eq. (32) are typically opposite to each other in their effects on the work function and surface exchange-correlation energy of jellium.

We have also calculated the energies of jellium spheres with LSD, PBE GGA, and TPSS and its predecessor PKZB meta-GGAs. Typically, while PBE energies are too low for spheres with more than about two electrons, LSD and TPSS are accurate there, up to 106 electrons. Curvature energies are reduced substantially as we pass from LSD to PBE to TPSS. Finally, we have shown that the linear response of bulk jellium (to perturbations with wave vectors less than twice the Fermi wave vector) is reasonably described by all the functionals considered here.

As we climb the ladder of nonempirical density functional approximations from LSD to GGA to meta-GGA, there is a steady and dramatic improvement in atomization energies. ${ }^{19,73}$ Surface energies worsen ${ }^{10-16,22}$ from LSD to PBE GGA (and other popular GGAs) due to an imperfect error cancellation between exchange and correlation, but this can be corrected in any of the three ways: (1) by transferring ${ }^{74}$ the needed correction from jellium to real systems, (2) by using GGAs designed specifically for solids (and not for free atoms), ${ }^{37-39}$ or (3) by climbing up further to the TPSS meta-GGA. The third way adds little in computational cost, ${ }^{19,73}$ even at full self-consistency, and seems worthy of further testing and possible refinement.

The jellium model itself remains useful as a testing ground for density functionals. Although some of its properties become unphysical as one moves away from the bulk density at which jellium is stable $\left(r_{s} \approx 4\right.$, roughly the valence density of sodium), this problem can also be fixed inexpensively via the stabilized jellium model. ${ }^{65,75}$

\section{ACKNOWLEDGMENTS}

This work was supported by the NSF under Grants No. DMR-0135678 (J.P.P. and J.T.) and No. DMR-0501588 (J.P.P.), by DOE under Contract No. DE-AC52-06NA25396 and Grant No. LDRD-PRD X9KU at LANL (J.T.), and by the Deutsche Forschungsgemeinschaft (S.K.).
${ }^{1}$ N. D. Lang and W. Kohn, Phys. Rev. B 1, 4555 (1970).

${ }^{2}$ W. Kohn and L. J. Sham, Phys. Rev. 140, A1133 (1965).

${ }^{3}$ J. P. Perdew and S. Kurth, in A Primer in Density Functional Theory, Lecture Notes in Physics Vol. 620, edited by C. Fiolhais, F. Nogueira, and M. Marques (Springer, Berlin, 2003).

${ }^{4}$ W. Kohn, Rev. Mod. Phys. 71, 1253 (1999).

${ }^{5}$ G. D. Mahan, Phys. Rev. B 12, 5585 (1975).

${ }^{6}$ J. P. Perdew and R. Monnier, Phys. Rev. Lett. 37, 1286 (1976).

${ }^{7}$ J. P. Perdew, Phys. Rev. B 16, 1525 (1977).

${ }^{8}$ R. Monnier and J. P. Perdew, Phys. Rev. B 17, 2595 (1978).

${ }^{9}$ R. L. Kautz and B. B. Schwartz, Phys. Rev. B 14, 2017 (1976).

${ }^{10}$ J. M. Pitarke and J. P. Perdew, Phys. Rev. B 67, 045101 (2003).

${ }^{11}$ V. N. Staroverov, G. E. Scuseria, J. Tao, and J. P. Perdew, Phys. Rev. B 69, 075102 (2004).

${ }^{12}$ L. A. Constantin, J. P. Perdew, and J. Tao, Phys. Rev. B 73, 205104 (2006).

${ }^{13}$ J. M. Pitarke, L. A. Constantin, and J. P. Perdew, Phys. Rev. B 74, 045121 (2006).

${ }^{14}$ B. Wood, N. D. M. Hine, W. M. C. Foulkes, and P. GarcíaGonzález, Phys. Rev. B 76, 035403 (2007).

${ }^{15}$ D. K. Yu, H. P. Bonzel, and M. Scheffler, Phys. Rev. B 74, 115408 (2006).

${ }^{16}$ L. A. Constantin, J. M. Pitarke, J. F. Dobson, A. Garcia-Lekue, and J. P. Perdew, Phys. Rev. Lett. 100, 036401 (2008).

${ }^{17}$ J. P. Perdew and Y. Wang, Phys. Rev. B 33, 8800 (1986); J. P. Perdew, ibid. 33, 8822 (1986); J. P. Perdew, ibid. 34, 7406(E) (1986).

${ }^{18}$ J. P. Perdew, K. Burke, and M. Ernzerhof, Phys. Rev. Lett. 77, 3865 (1996).

${ }^{19}$ V. N. Staroverov, G. E. Scuseria, J. Tao, and J. P. Perdew, J. Chem. Phys. 119, 12129 (2003); 121, 11507(E) (2004).

${ }^{20}$ S. Kurth, J. P. Perdew, and P. Blaha, Int. J. Quantum Chem. 75, 889 (1999).

${ }^{21}$ J. P. Perdew, Phys. Rev. Lett. 55, 1665 (1985).
${ }^{22}$ J. P. Perdew, S. Kurth, A. Zupan, and P. Blaha, Phys. Rev. Lett. 82, 2544 (1999)

${ }^{23}$ J. Tao, J. P. Perdew, V. N. Staroverov, and G. E. Scuseria, Phys. Rev. Lett. 91, 146401 (2003).

${ }^{24}$ P. S. Svendsen and U. von Barth, Phys. Rev. B 54, 17402 (1996).

${ }^{25}$ C. Adamo, M. Ernzerhof, and G. E. Scuseria, J. Chem. Phys. 112, 2643 (2000).

${ }^{26}$ A. D. Rabuck and G. E. Scuseria, Theor. Chem. Acc. 104, 439 (2000).

${ }^{27}$ J. P. Perdew, J. Tao, V. N. Staroverov, and G. E. Scuseria, J. Chem. Phys. 120, 6898 (2004).

${ }^{28}$ J. Tao, J. P. Perdew, A. Ruzsinszky, G. E. Scuseria, G. I. Csonka, and V. N. Staroverov, Philos. Mag. 87, 1071 (2007).

${ }^{29}$ V. N. Staroverov, G. E. Scuseria, J. P. Perdew, J. Tao, and E. R. Davidson, Phys. Rev. A 70, 012502 (2004).

${ }^{30}$ M. Seidl and J. P. Perdew, Phys. Rev. B 50, 5744 (1994).

${ }^{31}$ P. Ziesche, J. Tao, M. Seidl, and J. P. Perdew, Int. J. Quantum Chem. 77, 819 (2000).

${ }^{32}$ M. Seidl, J. P. Perdew, and M. Levy, Phys. Rev. A 59, 51 (1999).

${ }^{33}$ M. Seidl, J. P. Perdew, and S. Kurth, Phys. Rev. A 62, 012502 (2000).

${ }^{34}$ L. Miglio, M. P. Tosi, and N. H. March, Surf. Sci. 111, 119 (1981).

${ }^{35}$ J. P. Perdew and K. Schmidt, in Density Functional Theory and Its Application to Materials, edited by V. Van Doren, C. Van Alsenoy, and P. Geerlings, (AIP, Melville, New York, 2001).

${ }^{36}$ J. P. Perdew, A. Ruzsinszky, J. Tao, V. N. Staroverov, G. E. Scuseria, and G. I. Csonka, J. Chem. Phys. 123, 062201 (2005).

${ }^{37}$ R. Armiento and A. E. Mattsson, Phys. Rev. B 72, 085108 (2005).

${ }^{38}$ Z. Wu and R. E. Cohen, Phys. Rev. B 73, 235116 (2006).

${ }^{39}$ J. P. Perdew, A. Ruzsinszky, G. I. Csonka, O. A. Vydrov, G. E. 
Scuseria, L. A. Constantin, X. Zhou, and K. Burke, Phys. Rev. Lett. 100, 136406 (2008).

${ }^{40}$ G. L. Oliver and J. P. Perdew, Phys. Rev. A 20, 397 (1979).

${ }^{41}$ M. Levy and J. P. Perdew, Phys. Rev. A 32, 2010 (1985).

${ }^{42}$ J. P. Perdew, J. Tao, and S. Kümmel, in Electron Correlation Methodology, ACS Symposium Series Vol. 958, edited by A. K. Wilson and K. A. Peterson (Oxford University Press, New York, 2007).

${ }^{43}$ J. P. Perdew and Y. Wang, Phys. Rev. B 45, 13244 (1992).

${ }^{44}$ J. P. Perdew, K. Burke, and Y. Wang, Phys. Rev. B 54, 16533 (1996).

${ }^{45}$ Y. Wang and J. P. Perdew, Phys. Rev. B 43, 8911 (1991).

${ }^{46}$ J. Tao, J. Chem. Phys. 115, 3519 (2001).

${ }^{47}$ L. M. Almeida, J. P. Perdew, and C. Fiolhais, Phys. Rev. B 66, 075115 (2002).

${ }^{48}$ S.-K. Ma and K. A. Brueckner, Phys. Rev. 165, 18 (1968).

${ }^{49}$ D. J. W. Geldart and M. Rasolt, Phys. Rev. B 13, 1477 (1976).

${ }^{50}$ D. C. Langreth and J. P. Perdew, Phys. Rev. B 21, 5469 (1980).

${ }^{51}$ M. Swart, A. R. Groenhof, A. W. Ehlers, and K. Lammertsma, J. Phys. Chem. 108, 5479 (2004).

${ }^{52}$ S. Zein, S. H. Borshch, P. Fleurat-Lessard, M. E. Casida, and H. Chermette, J. Chem. Phys. 126, 014105 (2007).

${ }^{53} \mathrm{M}$. Swart (private communication).

${ }^{54}$ J. Bardeen, Phys. Rev. 49, 653 (1936).

${ }^{55}$ I. D. Moore and N. H. March, Ann. Phys. (N.Y.) 97, 136 (1976).

${ }^{56}$ L. Pollack and J. P. Perdew, J. Phys.: Condens. Matter 12, 1239 (2000).

${ }^{57}$ Z. Yan, J. P. Perdew, and S. Kurth, Phys. Rev. B 61, 16430 (2000).

${ }^{58}$ V. Sahni, J. Gruenebaum, and J. P. Perdew, Phys. Rev. B 26, 4371 (1982).
${ }^{59}$ F. Sottile and P. Ballone, Phys. Rev. B 64, 045105 (2001).

${ }^{60}$ G. Ortiz and P. Ballone, Phys. Rev. B 50, 1391 (1994).

${ }^{61}$ D. M. Ceperley and B. J. Alder, Phys. Rev. Lett. 45, 566 (1980).

${ }^{62}$ J. P. Perdew, Y. Wang, and E. Engel, Phys. Rev. Lett. 66, 508 (1991).

${ }^{63}$ See EPAPS Document No. E-PRBMDO-77-005824 for a detailed tabulation of deviations from corrected fixed-node DQMC values [Ref. 59 and Eq. (40)] of the total energies per electron for individual jellium clusters. For more information on EPAPS, see http://www.aip.org/pubservs/epaps.html.

${ }^{64}$ Q. M. Hu, K. Reuter, and M. Scheffler, Phys. Rev. Lett. 99, 169903(E) (2007).

${ }^{65}$ P. Ziesche, J. P. Perdew, and C. Fiolhais, Phys. Rev. B 49, 7916 (1994).

${ }^{66}$ W. A. Harrison, Pseudopotentials in the Theory of Metals (Benjamin, New York, 1966).

${ }^{67}$ J. P. Perdew and T. Datta, Phys. Status Solidi B 102, 283 (1980).

${ }^{68}$ M. Brack, B. K. Jennings, and Y. H. Chu, Phys. Lett. 65B, 1 (1976).

${ }^{69}$ W. Jones and W. H. Young, J. Phys. C 4, 1322 (1971).

${ }^{70}$ P. R. Antoniewicz and L. Kleinman, Phys. Rev. B 31, 6779 (1985).

${ }^{71}$ S. Moroni, D. M. Ceperley, and G. Senatore, Phys. Rev. Lett. 75, 689 (1995).

${ }^{72}$ C. F. Richardson and N. W. Ashcroft, Phys. Rev. B 50, 8170 (1994).

${ }^{73}$ F. Furche and J. P. Perdew, J. Chem. Phys. 124, 044103 (2006).

${ }^{74}$ T. R. Mattsson and A. E. Mattsson, Phys. Rev. B 66, 214110 (2002).

${ }^{75}$ J. P. Perdew, H. Q. Tran, and E. D. Smith, Phys. Rev. B 42, 11627 (1990). 